\title{
Relations for generalized Fibonacci and Tribonacci sequences
}

\author{
Robert Frontczak * \\ Landesbank Baden-Württemberg \\ Am Hauptbahnhof 2, 70173 Stuttgart, Germany \\ e-mail: robert.frontczak@lbbw. de
}

Received: 9 September $2018 \quad$ Revised: 20 February $2019 \quad$ Accepted: 23 February 2019

\begin{abstract}
In this article, we are concerned with connections between generalized Fibonacci and Tribonacci sequences. The identities we derive are of convolution type. As particular examples, we state several identities between Fibonacci and Tribonacci numbers, Fibonacci and TribonacciLucas numbers, Lucas and Tribonacci numbers and Lucas and Tribonacci-Lucas numbers, respectively. Our results provide extensions of some recently obtained identities.
\end{abstract}

Keywords: Generating function, Fibonacci number, Tribonacci number.

2010 Mathematics Subject Classification: 11B37, 11B39.

\section{Introduction and preliminaries}

Let $\left(u_{n}\right)_{n \geq 0}$ be a generalized Fibonacci sequence, i.e., a second-order recurrence given by

$$
u_{n}=u_{n-1}+u_{n-2}, \quad n \geq 2
$$

where $u_{0}$ and $u_{1}$ are arbitrary numbers not both being zero. Let also $\left(v_{n}\right)_{n \geq 0}$ be a generalized Tribonacci sequence, i.e., a third-order recurrence defined by

$$
v_{n}=v_{n-1}+v_{n-2}+v_{n-3}, \quad n \geq 3,
$$

* Disclaimer: Statements and conclusions made in this article are entirely those of the author. They do not necessarily reflect the views of LBBW. 
where $v_{0}, v_{1}$ and $v_{2}$ are arbitrary numbers not all being zero. When $u_{0}=0$ and $u_{1}=1$, then $u_{n}=F_{n}$ is the Fibonacci sequence (A000045 in [8]) and when $u_{0}=2$ and $u_{1}=1$, then $u_{n}=L_{n}$ is the Lucas sequence (A000032 in [8]). Also, when $v_{0}=0, v_{1}=v_{2}=1$, then $v_{n}=T_{n}$ is the Tribonacci sequence, whereas $v_{0}=3, v_{1}=1$ and $v_{2}=3$ gives $v_{n}=K_{n}$ the Tribonacci-Lucas sequence (sequences A000073 and A001644 in the OEIS [8], respectively). The most recent articles about generalized Fibonacci and Tribonacci numbers are the articles [1, 2, 3, 4] and [7].

Despite the fact that both sequences coexist for more than fifty years and there is a rich literature on both classes, they have been studied separately until now. The article [5] lists the first few connecting identities. In the present study, we explore some more connections between these popular sequences.

We will use the structure of the ordinary generating functions to prove our results. The generating functions for the two generalized sequences and their respective even and odd indexed companions are stated below:

$$
\begin{gathered}
f_{u_{n}}(x)=\sum_{n=0}^{\infty} u_{n} x^{n}=\frac{u_{0}+\left(u_{1}-u_{0}\right) x}{1-x-x^{2}} \\
f_{u_{2 n}}(x)=\sum_{n=0}^{\infty} u_{2 n} x^{n}=\frac{u_{0}+\left(u_{1}-2 u_{0}\right) x}{1-3 x+x^{2}} \\
f_{u_{2 n+1}}(x)=\sum_{n=0}^{\infty} u_{2 n+1} x^{n}=\frac{u_{1}+\left(u_{0}-u_{1}\right) x}{1-3 x+x^{2}} \\
f_{v_{n}}(x)=\sum_{n=0}^{\infty} v_{n} x^{n}=\frac{v_{0}+\left(v_{1}-v_{0}\right) x+\left(v_{2}-v_{1}-v_{0}\right) x^{2}}{1-x-x^{2}-x^{3}} \\
f_{v_{2 n}}(x)=\sum_{n=0}^{\infty} v_{2 n} x^{n}=\frac{v_{0}+\left(v_{2}-3 v_{0}\right) x+\left(2 v_{1}-v_{2}\right) x^{2}}{1-3 x-x^{2}-x^{3}}
\end{gathered}
$$

and

$$
f_{v_{2 n+1}}(x)=\sum_{n=0}^{\infty} v_{2 n+1} x^{n}=\frac{v_{1}+\left(v_{2}-2 v_{1}+v_{0}\right) x+\left(v_{2}-v_{1}-v_{0}\right) x^{2}}{1-3 x-x^{2}-x^{3}} .
$$

The expressions follow from more or less routine calculations. The functions for $u_{n}$ are derived in [6], these for $v_{n}$ come from [4].

\section{Basic relations between $u_{n}$ and $v_{n}$}

The main results of this section reveal three fundamental relations between generalized Fibonacci and Tribonacci numbers. We present our findings in three separate theorems. We note that, in what follows, we will use the standard convention that $\sum_{k=0}^{n} a_{k}=0$ for $n<0$.

Theorem 2.1. Let $\left(u_{n}\right)_{n \geq 0}$ and $\left(v_{n}\right)_{n \geq 0}$ denote the generalized Fibonacci and Tribonacci sequences, respectively. Then, for each $n \geq 2$, we have the following identity:

$$
u_{0} v_{n}=\left(u_{0}-u_{1}\right) v_{n-1}+v_{0} u_{n}+\left(v_{1}-v_{0}\right) u_{n-1}+\left(v_{2}-v_{1}-v_{0}\right) u_{n-2}+\sum_{k=0}^{n-3} u_{k} v_{n-3-k} .
$$


Proof: From (1.3) we deduce that

$$
\frac{u_{0}+\left(u_{1}-u_{0}\right) x}{f_{u_{n}}(x)}=1-x-x^{2} .
$$

Hence, it follows that

$$
\frac{u_{0}+\left(u_{1}-u_{0}\right) x-x^{3} f_{u_{n}}(x)}{f_{u_{n}}(x)}=1-x-x^{2}-x^{3} .
$$

This yields the relation

$$
f_{v_{n}}(x)=\left(v_{0}+\left(v_{1}-v_{0}\right) x+\left(v_{2}-v_{1}-v_{0}\right) x^{2}\right) \frac{f_{u_{n}}(x)}{u_{0}+\left(u_{1}-u_{0}\right) x-x^{3} f_{u_{n}}(x)},
$$

or equivalently,

$$
u_{0} f_{v_{n}}(x)-v_{0} f_{u_{n}}(x)=\left(u_{0}-u_{1}\right) x f_{v_{n}}(x)+\left(v_{1}-v_{0}\right) x f_{u_{n}}(x)+\left(v_{2}-v_{1}-v_{0}\right) x^{2} f_{u_{n}}(x)+x^{3} f_{u_{n}}(x) f_{v_{n}}(x) .
$$

The LHS of the last equation is

$$
L H S=\sum_{n=1}^{\infty}\left(u_{0} v_{n}-v_{0} u_{n}\right) x^{n} .
$$

For the RHS we have

$$
\begin{aligned}
\text { RHS }=\left(u_{0}-u_{1}\right) \sum_{n=1}^{\infty} v_{n-1} x^{n}+\left(v_{1}-v_{0}\right) \sum_{n=1}^{\infty} u_{n-1} x^{n} \\
\quad+\left(v_{2}-v_{1}-v_{0}\right) \sum_{n=2}^{\infty} u_{n-2} x^{n}+\sum_{n=3}^{\infty} \sum_{k=0}^{n-3} u_{k} v_{n-3-k} x^{n} .
\end{aligned}
$$

Gathering terms and comparing the coefficients for $x^{n}$ proves the formula.

When $u_{n}=F_{n}$ and $v_{n}=T_{n}$, then equation (2.1) simplifies to

$$
T_{n}=F_{n}+\sum_{k=0}^{n-2} F_{k} T_{n-2-k}
$$

When $u_{n}=L_{n}$ and $v_{n}=T_{n}$, then equation (2.1) becomes

$$
2 T_{n}=T_{n-1}+L_{n-1}+\sum_{k=0}^{n-3} L_{k} T_{n-3-k} .
$$

When $u_{n}=F_{n}$ and $v_{n}=K_{n}$, then we get

$$
K_{n-1}=3 F_{n}-2 F_{n-1}-F_{n-2}+\sum_{k=0}^{n-3} F_{k} K_{n-3-k}=L_{n-1}+\sum_{k=0}^{n-3} F_{k} K_{n-3-k} .
$$

Finally, when $u_{n}=L_{n}$ and $v_{n}=K_{n}$, then

$$
2 K_{n}=K_{n-1}+L_{n}+L_{n-2}+\sum_{k=0}^{n-3} L_{k} K_{n-3-k} .
$$

The four special cases have been derived recently in the article [5]. The next identity gives us a basic relation between the even indexed sequences $\left(u_{2 n}\right)_{n \geq 0}$ and $\left(v_{2 n}\right)_{n \geq 0}$. 
Theorem 2.2. For $n \geq 2$ the following identity holds:

$$
\begin{aligned}
u_{0} v_{2 n}=\left(2 u_{0}\right. & \left.-u_{1}\right) v_{2 n-2}+v_{0} u_{2 n}+\left(v_{2}-3 v_{0}\right) u_{2 n-2}+\left(2 v_{1}-v_{2}+2 v_{0}\right) u_{2 n-4} \\
& +\sum_{k=0}^{n-3} u_{2 k}\left(2 v_{2(n-2-k)}+v_{2(n-3-k)}\right) .
\end{aligned}
$$

Proof: From (1.4) we have

$$
\frac{u_{0}+\left(u_{1}-2 u_{0}\right) x-2 x^{2} f_{u_{2 n}}(x)-x^{3} f_{u_{2 n}}(x)}{f_{u_{2 n}}(x)}=1-3 x-x^{2}-x^{3} .
$$

Combining this relation with the generating function (1.7) yields

$$
\begin{aligned}
u_{0} f_{v_{2 n}}(x)-v_{0} f_{u_{2 n}}(x)=( & \left.2 u_{0}-u_{1}\right) x f_{v_{2 n}}(x)+\left(v_{2}-3 v_{0}\right) x f_{u_{2 n}}(x)+\left(2 v_{1}-v_{2}\right) x^{2} f_{u_{2 n}}(x) \\
& +2 x^{2} f_{u_{2 n}}(x) f_{v_{2 n}}(x)+x^{3} f_{u_{2 n}}(x) f_{v_{2 n}}(x) .
\end{aligned}
$$

The remaining part of the proof is as in Theorem 2.1.

As specific examples we can state the following identities:

1. $u_{2 n}=F_{2 n}$ and $v_{2 n}=T_{2 n}$ :

$$
T_{2 n}=F_{2 n}+F_{2 n-2}+\sum_{k=0}^{n-2} F_{2 k}\left(2 T_{2(n-1-k)}+T_{2(n-2-k)}\right) .
$$

2. $u_{2 n}=L_{2 n}$ and $v_{2 n}=T_{2 n}$ :

$$
2 T_{2 n}=3 T_{2 n-2}+L_{2 n-2}+L_{2 n-4}+\sum_{k=0}^{n-3} L_{2 k}\left(2 T_{2(n-2-k)}+T_{2(n-3-k)}\right) .
$$

3. $u_{2 n}=F_{2 n}$ and $v_{2 n}=K_{2 n}$ :

$$
K_{2 n-2}=3 F_{2 n}-6 F_{2 n-2}+5 F_{2 n-4}+\sum_{k=0}^{n-3} F_{2 k}\left(2 K_{2(n-2-k)}+K_{2(n-3-k)}\right) .
$$

4. $u_{2 n}=L_{2 n}$ and $v_{2 n}=K_{2 n}$ :

$$
2 K_{2 n}=3 K_{2 n-2}+3 L_{2 n}-6 L_{2 n-2}+5 L_{2 n-4}+\sum_{k=0}^{n-3} L_{2 k}\left(2 K_{2(n-2-k)}+K_{2(n-3-k)}\right) .
$$

Finally, we present an identity for the odd indexed numbers $\left(u_{2 n+1}\right)_{n \geq 0}$ and $\left(v_{2 n+1}\right)_{n \geq 0}$.

Theorem 2.3. For $n \geq 2$ the following identity holds:

$$
\begin{gathered}
u_{1} v_{2 n+1}=\left(u_{1}-u_{0}\right) v_{2 n-1}+v_{1} u_{2 n+1}+\left(v_{2}-2 v_{1}+v_{0}\right) u_{2 n-1}+\left(v_{2}+v_{1}-v_{0}\right) u_{2 n-3} \\
+\sum_{k=0}^{n-3} u_{2 k+1}\left(2 v_{2(n-2-k)+1}+v_{2(n-3-k)+1}\right) .
\end{gathered}
$$


Proof: The stated formula is essentially a consequence of the relation

$$
\begin{aligned}
u_{1} f_{v_{2 n+1}}(x)-v_{1} f_{u_{2 n+1}}(x)= & \left(u_{1}-u_{0}\right) x f_{v_{2 n+1}}(x)+\left(v_{2}-2 v_{1}+v_{0}\right) x f_{u_{2 n+1}}(x) \\
& +\left(v_{2}-v_{1}-v_{0}\right) x^{2} f_{u_{2 n+1}}(x)+2 x^{2} f_{u_{2 n+1}}(x) f_{v_{2 n+1}}(x) \\
& +x^{3} f_{u_{2 n+1}}(x) f_{v_{2 n+1}}(x),
\end{aligned}
$$

which can be derived using (1.5) and (1.8).

Examples are:

1. $u_{2 n+1}=F_{2 n+1}$ and $v_{2 n+1}=T_{2 n+1}$ :

$$
T_{2 n+1}=T_{2 n-1}+F_{2 n+1}-F_{2 n-1}+2 F_{2 n-3}+\sum_{k=0}^{n-3} F_{2 k+1}\left(2 T_{2(n-2-k)+1}+T_{2(n-3-k)+1}\right) .
$$

2. $u_{2 n+1}=L_{2 n+1}$ and $v_{2 n+1}=T_{2 n+1}$ :

$$
T_{2 n+1}=-T_{2 n-1}+L_{2 n+1}-L_{2 n-1}+2 L_{2 n-3}+\sum_{k=0}^{n-3} L_{2 k+1}\left(2 T_{2(n-2-k)+1}+T_{2(n-3-k)+1}\right) .
$$

3. $u_{2 n+1}=F_{2 n+1}$ and $v_{2 n+1}=K_{2 n+1}$ :

$$
K_{2 n+1}=K_{2 n-1}+F_{2 n+1}+4 F_{2 n-1}+F_{2 n-3}+\sum_{k=0}^{n-3} F_{2 k+1}\left(2 K_{2(n-2-k)+1}+K_{2(n-3-k)+1}\right) .
$$

4. $u_{2 n+1}=L_{2 n+1}$ and $v_{2 n+1}=K_{2 n+1}$ :

$$
K_{2 n+1}=-K_{2 n-1}+L_{2 n+1}+4 L_{2 n-1}+L_{2 n-3}+\sum_{k=0}^{n-3} L_{2 k+1}\left(2 K_{2(n-2-k)+1}+K_{2(n-3-k)+1}\right) \text {. }
$$

Combining the last equations for $T_{2 n+1}$ and $K_{2 n+1}$, respectively, results in the following corollary:

Corollary 2.4. For $n \geq 2$ it holds that

$$
\begin{aligned}
2 T_{2 n+1}=( & \left.L_{2 n+1}+F_{2 n+1}\right)-\left(L_{2 n-1}+F_{2 n-1}\right)+2\left(L_{2 n-3}+F_{2 n-3}\right) \\
& +\sum_{k=0}^{n-3}\left(L_{2 k+1}+F_{2 k+1}\right)\left(2 T_{2(n-2-k)+1}+T_{2(n-3-k)+1}\right)
\end{aligned}
$$

and

$$
\begin{aligned}
2 T_{2 n-1}=( & \left.L_{2 n+1}-F_{2 n+1}\right)-\left(L_{2 n-1}-F_{2 n-1}\right)+2\left(L_{2 n-3}-F_{2 n-3}\right) \\
& +\sum_{k=0}^{n-3}\left(L_{2 k+1}-F_{2 k+1}\right)\left(2 T_{2(n-2-k)+1}+T_{2(n-3-k)+1}\right) .
\end{aligned}
$$


Similarly,

$$
\begin{aligned}
2 K_{2 n+1}= & \left(L_{2 n+1}+F_{2 n+1}\right)+4\left(L_{2 n-1}+F_{2 n-1}\right)+\left(L_{2 n-3}+F_{2 n-3}\right) \\
& +\sum_{k=0}^{n-3}\left(L_{2 k+1}+F_{2 k+1}\right)\left(2 K_{2(n-2-k)+1}+K_{2(n-3-k)+1}\right),
\end{aligned}
$$

and

$$
\begin{aligned}
2 K_{2 n-1}= & \left(L_{2 n+1}-F_{2 n+1}\right)+4\left(L_{2 n-1}-F_{2 n-1}\right)+\left(L_{2 n-3}-F_{2 n-3}\right) \\
& +\sum_{k=0}^{n-3}\left(L_{2 k+1}-F_{2 k+1}\right)\left(2 K_{2(n-2-k)+1}+K_{2(n-3-k)+1}\right) .
\end{aligned}
$$

\section{More advanced relations}

This section contains further relations for $u_{n}$ and $v_{n}$ that can be derived in a straightforward manner from the last section. To preserve an easier to comprehend and concise presentation, from now on, we will restrict the list of examples to the pairs $\left(u_{n}=F_{n}, v_{n}=T_{n}\right)$ and $\left(u_{n}=\right.$ $L_{n}, v_{n}=T_{n}$ ) only.

Theorem 3.1. For $n \geq 1$ the following identity holds:

$$
\begin{aligned}
u_{0} \sum_{k=0}^{n} v_{k} v_{n-k}= & v_{0} \sum_{k=0}^{n} v_{k} u_{n-k}+\sum_{k=0}^{n-1} v_{n-1-k}\left(\left(u_{0}-u_{1}\right) v_{k}+\left(v_{1}-v_{0}\right) u_{k}\right) \\
& +\left(v_{2}-v_{1}-v_{0}\right) \sum_{k=0}^{n-2} u_{k} v_{n-2-k}+\sum_{k_{1}+k_{2}+k_{3}=n-3} u_{k_{1}} v_{k_{2}} v_{k_{3}} .
\end{aligned}
$$

Proof: We start with equation (2.2). Multiplying both sides by $f_{v_{n}}(x)$ gives

$$
\begin{aligned}
f_{v_{n}}(x)\left(u_{0} f_{v_{n}}(x)-v_{0} f_{u_{n}}(x)\right)= & \left(u_{0}-u_{1}\right) x f_{v_{n}}^{2}(x)+\left(v_{1}-v_{0}\right) x f_{u_{n}}(x) f_{v_{n}}(x) \\
& +\left(v_{2}-v_{1}-v_{0}\right) x^{2} f_{u_{n}}(x) f_{v_{n}}(x)+x^{3} f_{u_{n}}(x) f_{v_{n}}^{2}(x) .
\end{aligned}
$$

The LHS equals

$$
L H S=\sum_{n=1}^{\infty}\left(\sum_{k=0}^{n} v_{k}\left(u_{0} v_{n-k}-v_{0} u_{n-k}\right)\right) x^{n} .
$$

The RHS is

$$
\begin{aligned}
\text { RHS }= & \left(u_{0}-u_{1}\right) \sum_{n=1}^{\infty}\left(\sum_{k=0}^{n-1} v_{k} v_{n-1-k}\right) x^{n}+\left(v_{1}-v_{0}\right) \sum_{n=1}^{\infty}\left(\sum_{k=0}^{n-1} u_{k} v_{n-1-k}\right) x^{n} \\
& +\left(v_{2}-v_{1}-v_{0}\right) \sum_{n=2}^{\infty}\left(\sum_{k=0}^{n-2} u_{k} v_{n-2-k}\right) x^{n}+\sum_{n=3}^{\infty}\left(\sum_{k_{1}+k_{2}+k_{3}=n-3} u_{k_{1}} v_{k_{2}} v_{k_{3}}\right) x^{n} .
\end{aligned}
$$

Gathering terms and comparing the coefficients for $x^{n}$ establishes the stated relation. 
When $u_{n}=F_{n}$ and $v_{n}=T_{n}$, then for $n \geq 1$ :

$$
\sum_{k=0}^{n} T_{k} T_{n-k}=\sum_{k=0}^{n} F_{k} T_{n-k}+\sum_{\substack{k_{1}+k_{2}+k_{3}=n-2 \\ k_{1}, k_{2}, k_{3} \geq 1}} F_{k_{1}} T_{k_{2}} T_{k_{3}}
$$

When $u_{n}=L_{n}$ and $v_{n}=T_{n}$, then for $n \geq 1$ :

$$
2 \sum_{k=0}^{n} T_{k} T_{n-k}=\sum_{k=0}^{n-1}\left(T_{k}+L_{k}\right) T_{n-1-k}+\sum_{\substack{k_{1}+k_{2}+k_{3}=n-3 \\ k_{1} \geq 0, k_{2}, k_{3} \geq 1}} L_{k_{1}} T_{k_{2}} T_{k_{3}} .
$$

Theorem 3.2. For $n \geq 1$ the following identity holds:

$$
\begin{aligned}
u_{0} \sum_{k=0}^{n} u_{k} v_{n-k}= & v_{0} \sum_{k=0}^{n} u_{k} u_{n-k}+\sum_{k=0}^{n-1} u_{k}\left(\left(u_{0}-u_{1}\right) v_{n-1-k}+\left(v_{1}-v_{0}\right) u_{n-1-k}\right) \\
& +\left(v_{2}-v_{1}-v_{0}\right) \sum_{k=0}^{n-2} u_{k} u_{n-2-k}+\sum_{k_{1}+k_{2}+k_{3}=n-3} u_{k_{1}} u_{k_{2}} v_{k_{3}} .
\end{aligned}
$$

Proof: The relation follows from multiplying equation (2.2) by $f_{u_{n}}(x)$, writing in terms of power series, and collecting terms. We omit the details.

Theorem 3.2 is a generalization of results from [5], where a detailed treatment of the pairs $\left(u_{n}=F_{n}, v_{n}=T_{n}\right),\left(u_{n}=L_{n}, v_{n}=T_{n}\right),\left(u_{n}=F_{n}, v_{n}=K_{n}\right)$, and $\left(u_{n}=L_{n}, v_{n}=K_{n}\right)$ is presented.

For the even indexed sequences $\left(u_{2 n}\right)_{n \geq 0}$ and $\left(v_{2 n}\right)_{n \geq 0}$ we have the following convolutions:

Theorem 3.3. For $n \geq 1$ the following identity holds:

$$
\begin{aligned}
u_{0} \sum_{k=0}^{n} v_{2 k} v_{2(n-k)}= & v_{0} \sum_{k=0}^{n} v_{2 k} u_{2(n-k)}+\sum_{k=0}^{n-1} v_{2(n-1-k)}\left(\left(2 u_{0}-u_{1}\right) v_{2 k}+\left(v_{2}-3 v_{0}\right) u_{2 k}\right) \\
& +\left(2 v_{1}-v_{2}\right) \sum_{k=0}^{n-2} u_{2 k} v_{2(n-2-k)}+2 \sum_{k_{1}+k_{2}+k_{3}=n-2} u_{2 k_{1}} v_{2 k_{2}} v_{2 k_{3}} \\
& +\sum_{k_{1}+k_{2}+k_{3}=n-3} u_{2 k_{1}} v_{2 k_{2}} v_{2 k_{3}} .
\end{aligned}
$$

Proof: The relation follows from multiplying equation (2.10) by $f_{v_{2 n}}(x)$, writing in terms of power series and collecting terms. We omit the details.

The special case when $u_{n}=F_{n}$ and $v_{n}=T_{n}$ can be stated as $(n \geq 1)$ :

$$
\begin{aligned}
\sum_{k=0}^{n} T_{2 k} T_{2(n+1-k)}= & \sum_{k=0}^{n} F_{2 k}\left(T_{2(n+1-k)}+T_{2(n-k)}\right)+2 \sum_{\substack{k_{1}+k_{2}+k_{3}=n \\
k_{1}, k_{2}, k_{3} \geq 1}} F_{2 k_{1}} T_{2 k_{2}} T_{2 k_{3}} \\
& +\sum_{\substack{k_{1}+k_{2}+k_{3}=n-1 \\
k_{1}, k_{2}, k_{3} \geq 1}} F_{2 k_{1}} T_{2 k_{2}} T_{2 k_{3}} .
\end{aligned}
$$


When $u_{n}=L_{n}$ and $v_{n}=T_{n}$, then we get for $n \geq 1$ :

$$
\begin{aligned}
& 2 \sum_{k=0}^{n} T_{2 k} T_{2(n-k)}=\sum_{k=0}^{n-2} L_{2 k}\left(T_{2(n-1-k)}+T_{2(n-2-k)}\right)+3 \sum_{k=0}^{n-2} T_{2 k} T_{2(n-1-k)} \\
& 2 \sum_{\substack{k_{1}+k_{2}+k_{3}=n-2 \\
k_{1} \geq 0, k_{2}, k_{3} \geq 1}} L_{2 k_{1}} T_{2 k_{2}} T_{2 k_{3}}+\sum_{\substack{k_{1}+k_{2}+k_{3}=n-3 \\
k_{1} \geq 0, k_{2}, k_{3} \geq 1}} L_{2 k_{1}} T_{2 k_{2}} T_{2 k_{3}} .
\end{aligned}
$$

Theorem 3.4. For $n \geq 1$ the following identity holds:

$$
\begin{aligned}
u_{0} \sum_{k=0}^{n} u_{2 k} v_{2(n-k)}= & v_{0} \sum_{k=0}^{n} u_{2 k} u_{2(n-k)}+\sum_{k=0}^{n-1} u_{2 k}\left(\left(2 u_{0}-u_{1}\right) v_{2(n-1-k)}+\left(v_{2}-3 v_{0}\right) u_{2(n-1-k)}\right) \\
& +\left(2 v_{1}-v_{2}\right) \sum_{k=0}^{n-2} u_{2 k} u_{2(n-2-k)}+2 \sum_{k_{1}+k_{2}+k_{3}=n-2} u_{2 k_{1}} u_{2 k_{2}} v_{2 k_{3}} \\
& +\sum_{k_{1}+k_{2}+k_{3}=n-3} u_{2 k_{1}} u_{2 k_{2}} v_{2 k_{3}} .
\end{aligned}
$$

Proof: The relation follows from multiplying equation (2.10) by $f_{u_{2 n}}(x)$, writing in terms of power series and collecting terms. We omit the details.

The special case when $u_{n}=F_{n}$ and $v_{n}=T_{n}$ is $(n \geq 1)$ :

$$
\begin{aligned}
\sum_{k=0}^{n} F_{2 k} T_{2(n+1-k)}= & \sum_{k=0}^{n} F_{2 k}\left(F_{2(n+1-k)}+F_{2(n-k)}\right)+2 \sum_{\substack{k_{1}+k_{2}+k_{3}=n \\
k_{1}, k_{2}, k_{3} \geq 1}} F_{2 k_{1}} F_{2 k_{2}} T_{2 k_{3}} \\
& +\sum_{\substack{k_{1}+k_{2}+k_{3}=n-1 \\
k_{1}, k_{2}, k_{3} \geq 1}} F_{2 k_{1}} F_{2 k_{2}} T_{2 k_{3}} .
\end{aligned}
$$

When $u_{n}=L_{n}$ and $v_{n}=T_{n}$, then we get for $n \geq 1$ :

$$
\begin{aligned}
& 2 \sum_{k=0}^{n} L_{2 k} T_{2(n-k)}=\sum_{k=0}^{n-1} L_{2 k}\left(3 T_{2(n-1-k)}+L_{2(n-1-k)}\right)+\sum_{k=0}^{n-2} L_{2 k} L_{2(n-2-k)} \\
& 2 \sum_{\substack{k \\
k_{1}+k_{2}+k_{3}=n-2 \\
k_{1}, k_{2} \geq 0, k_{3} \geq 1}} L_{2 k_{1}} L_{2 k_{2}} T_{2 k_{3}}+\sum_{\substack{k_{1}+k_{2}+k_{3}=n-3 \\
k_{1}, k_{2} \geq 0, k_{3} \geq 1}} L_{2 k_{1}} L_{2 k_{2}} T_{2 k_{3}} .
\end{aligned}
$$

We conclude this section with two convolutions for the odd indexed sequences $\left(u_{2 n+1}\right)_{n \geq 0}$ and $\left(v_{2 n+1}\right)_{n \geq 0}$. 
Theorem 3.5. For $n \geq 1$ the following identity holds:

$$
\begin{aligned}
u_{1} \sum_{k=0}^{n} v_{2 k+1} v_{2(n-k)+1}= & v_{1} \sum_{k=0}^{n} v_{2 k+1} u_{2(n-k)+1} \\
& +\sum_{k=0}^{n-1} v_{2 k+1}\left(\left(u_{1}-u_{0}\right) v_{2(n-1-k)+1}+\left(v_{2}-2 v_{1}-v_{0}\right) u_{2(n-1-k)+1}\right) \\
& +\left(v_{2}-v_{1}-v_{0}\right) \sum_{k=0}^{n-2} v_{2 k+1} u_{2(n-2-k)+1}+2 \sum_{k_{1}+k_{2}+k_{3}=n-2} u_{2 k_{1}+1} v_{2 k_{2}+1} v_{2 k_{3}+1} \\
& +\sum_{k_{1}+k_{2}+k_{3}=n-3} u_{2 k_{1}+1} v_{2 k_{2}+1} v_{2 k_{3}+1} .
\end{aligned}
$$

Proof: The relation follows from multiplying equation (2.16) by $f_{v_{2 n+1}}(x)$, writing in terms of power series, and collecting terms.

We state the special case when $u_{n}=F_{n}$ and $v_{n}=T_{n}$ as $(n \geq 0)$ :

$$
\begin{aligned}
& \sum_{k=0}^{n} T_{2 k+1}\left(T_{2(n+1-k)+1}-T_{2(n-k)+1}\right)=\sum_{k=0}^{n} T_{2 k+1}\left(F_{2(n+1-k)+1}-F_{2(n-k)+1}\right) \\
& \quad+2 \sum_{\substack{k=0 \\
k_{1}+k_{2}+k_{3}=n-1 \\
k_{1}, k_{2}, k_{3} \geq 0}} F_{2 k_{1}+1} T_{2 k_{2}+1} T_{2 k_{3}+1}+\sum_{\substack{k_{1}+k_{2}+k_{3}=n-2 \\
k_{1}, k_{2}, k_{3} \geq 0}} F_{2 k_{1}+1} T_{2 k_{2}+1} T_{2 k_{3}+1}
\end{aligned}
$$

When $u_{n}=L_{n}$ and $v_{n}=T_{n}$, then the relation becomes $(n \geq 0)$ :

$$
\begin{aligned}
& \sum_{k=0}^{n} T_{2 k+1}\left(T_{2(n+1-k)+1}+T_{2(n-k)+1}\right)=\sum_{k=0}^{n} T_{2 k+1}\left(L_{2(n+1-k)+1}-L_{2(n-k)+1}\right) \\
& \quad+2 \sum_{\substack{k_{1}+k_{2}+k_{3}=n-1 \\
k_{1}, k_{2}, k_{3} \geq 0}} L_{2 k_{1}+1} T_{2 k_{2}+1} T_{2 k_{3}+1}+\sum_{\substack{k_{1}+k_{2}+k_{3}=n-2 \\
k_{1}, k_{2}, k_{3} \geq 0}} L_{2 k_{1}+1} T_{2 k_{2}+1} T_{2 k_{3}+1}
\end{aligned} .
$$

Theorem 3.6. For $n \geq 1$ the following identity holds:

$$
\begin{aligned}
u_{1} \sum_{k=0}^{n} u_{2 k+1} v_{2(n-k)+1}= & v_{1} \sum_{k=0}^{n} u_{2 k+1} u_{2(n-k)+1} \\
& +\sum_{k=0}^{n-1} u_{2 k+1}\left(\left(u_{1}-u_{0}\right) v_{2(n-1-k)+1}+\left(v_{2}-2 v_{1}-v_{0}\right) u_{2(n-1-k)+1}\right) \\
& +\left(v_{2}-v_{1}-v_{0}\right) \sum_{k=0}^{n-2} u_{2 k+1} u_{2(n-2-k)+1}+2 \sum_{k_{1}+k_{2}+k_{3}=n-2} u_{2 k_{1}+1} u_{2 k_{2}+1} v_{2 k_{3}+1} \\
& +\sum_{k_{1}+k_{2}+k_{3}=n-3} u_{2 k_{1}+1} u_{2 k_{2}+1} v_{2 k_{3}+1} .
\end{aligned}
$$


Proof: The relation follows from multiplying equation (2.16) by $f_{u_{2 n+1}}(x)$, writing in terms of power series, and collecting terms.

When $u_{n}=F_{n}$ and $v_{n}=T_{n}$, then we get for $n \geq 0$ :

$$
\begin{aligned}
& \sum_{k=0}^{n} F_{2 k+1}\left(T_{2(n+1-k)+1}-T_{2(n-k)+1}\right)=\sum_{k=0}^{n} F_{2 k+1}\left(F_{2(n+1-k)+1}-F_{2(n-k)+1}\right) \\
& \quad+2 \sum_{\substack{k_{1}+k_{2}+k_{3}=n-1 \\
k_{1}, k_{2}, k_{3} \geq 0}} F_{2 k_{1}+1} F_{2 k_{2}+1} T_{2 k_{3}+1}+\sum_{\substack{k_{1}+k_{2}+k_{3}=n-2 \\
k_{1}, k_{2}, k_{3} \geq 0}} F_{2 k_{1}+1} F_{2 k_{2}+1} T_{2 k_{3}+1} .
\end{aligned}
$$

When $u_{n}=L_{n}$ and $v_{n}=T_{n}$, then we get for $n \geq 0$ :

$$
\begin{aligned}
& \sum_{k=0}^{n} L_{2 k+1}\left(T_{2(n+1-k)+1}+T_{2(n-k)+1}\right)=\sum_{k=0}^{n} L_{2 k+1}\left(L_{2(n+1-k)+1}-L_{2(n-k)+1}\right) \\
& \quad+2 \sum_{\substack{k_{1}+k_{2}+k_{3}=n-1 \\
k_{1}, k_{2}, k_{3} \geq 0}} L_{2 k_{1}+1} L_{2 k_{2}+1} T_{2 k_{3}+1}+\sum_{\substack{k_{1}+k_{2}+k_{3}=n-2 \\
k_{1}, k_{2}, k_{3} \geq 0}} L_{2 k_{1}+1} L_{2 k_{2}+1} T_{2 k_{3}+1} .
\end{aligned}
$$

\section{The general case}

From equations (2.2), (2.10), and (2.16) the general structure of the relations between the sequences $\left(u_{n}\right)_{n \geq 0}$ and $\left(v_{n}\right)_{n \geq 0}$ can be inferred. Let $m \geq 0$ be an integer. Then, for instance, multiplying (2.2) by $f_{v_{n}}^{m}(x)$ gives

$$
\begin{aligned}
u_{0} f_{v_{n}}^{m+1}(x)= & v_{0} f_{u_{n}}(x) f_{v_{n}}^{m}(x)+\left(u_{0}-u_{1}\right) x f_{v_{n}}^{m+1}(x)+\left(v_{1}-v_{0}\right) x f_{u_{n}}(x) f_{v_{n}}^{m}(x) \\
& +\left(v_{2}-v_{1}-v_{0}\right) x^{2} f_{u_{n}}(x) f_{v_{n}}^{m}(x)+x^{3} f_{u_{n}}(x) f_{v_{n}}^{m+1}(x) .
\end{aligned}
$$

Similarly, multiplying (2.2) by $f_{u_{n}}^{m}(x)$ gives

$$
\begin{aligned}
v_{0} f_{u_{n}}^{m+1}(x)= & u_{0} f_{v_{n}}(x) f_{u_{n}}^{m}(x)+\left(u_{1}-u_{0}\right) x f_{v_{n}}(x) f_{u_{n}}^{m}(x)+\left(v_{0}-v_{1}\right) x f_{u_{n}}^{m+1}(x) \\
& +\left(v_{0}+v_{1}-v_{2}\right) x^{2} f_{v u_{n}}^{m+1}(x)-x^{3} f_{v_{n}}(x) f_{u_{n}}^{m+1}(x) .
\end{aligned}
$$

From these relations it is straightforward to derive the following general identities, which we state in the next theorem:

Theorem 4.1. Let $m \geq 0$ be an integer. Then the following identities hold:

$$
\begin{aligned}
& u_{0} \sum_{k_{1}+k_{2}+\cdots+k_{m+1}=n} v_{k_{1}} v_{k_{2}} \cdots v_{k_{m+1}}=v_{0} \sum_{k_{1}+k_{2}+\cdots+k_{m+1}=n} u_{k_{1}} v_{k_{2}} \cdots v_{k_{m+1}} \\
& +\sum_{k_{1}+k_{2}+\cdots+k_{m+1}=n-1}\left(\left(u_{0}-u_{1}\right) v_{k_{1}} v_{k_{2}} \cdots v_{k_{m+1}}+\left(v_{1}-v_{0}\right) u_{k_{1}} v_{k_{2}} \cdots v_{k_{m+1}}\right) \\
& +\left(v_{2}-v_{1}-v_{0}\right) \sum_{k_{1}+k_{2}+\cdots+k_{m+1}=n-2} u_{k_{1}} v_{k_{2}} \cdots v_{k_{m+1}}+\sum_{k_{1}+k_{2}+\cdots+k_{m+2}=n-3} \sum_{k_{1}} v_{k_{2}} \cdots v_{k_{m+2}}
\end{aligned}
$$


and

$$
\begin{aligned}
& v_{0} \sum_{k_{1}+k_{2}+\cdots+k_{m+1}=n} u_{k_{1}} u_{k_{2}} \cdots u_{k_{m+1}}=u_{0} \sum_{k_{1}+k_{2}+\cdots+k_{m+1}=n} v_{k_{1}} u_{k_{2}} \cdots u_{k_{m+1}} \\
& +\sum_{k_{1}+k_{2}+\cdots+k_{m+1}=n-1}\left(\left(u_{1}-u_{0}\right) v_{k_{1}} u_{k_{2}} \cdots u_{k_{m+1}}+\left(v_{0}-v_{1}\right) u_{k_{1}} u_{k_{2}} \cdots u_{k_{m+1}}\right) \\
& +\left(v_{0}+v_{1}-v_{2}\right) \sum_{k_{1}+k_{2}+\cdots+k_{m+1}=n-2} u_{k_{1}} u_{k_{2}} \cdots u_{k_{m+1}}-\sum_{k_{1}+k_{2}+\cdots+k_{m+2}=n-3} v_{k_{1}} u_{k_{2}} \cdots u_{k_{m+2}} .
\end{aligned}
$$

From (4.3) with $u_{n}=F_{n}$ and $v_{n}=T_{n}$ we obtain

$$
\begin{aligned}
& \sum_{\substack{k_{1}+k_{2}+\cdots+k_{m+2}=n-2 \\
k_{1}, k_{2}, \ldots, k_{m+2} \geq 1}} F_{k_{1}} T_{k_{2}} \cdots T_{k_{m+2}}=\sum_{\substack{k_{1}+k_{2}+\cdots+k_{m+1}=n \\
k_{1}, k_{2}, \ldots, k_{m+1} \geq 1}} T_{k_{1}} T_{k_{2}} \cdots T_{k_{m+1}} \\
& -\sum_{\substack{k_{1}+k_{2}+\cdots+k_{m+1}=n \\
k_{1}, k_{2}, \ldots, k_{m+1} \geq 1}} F_{k_{1}} T_{k_{2}} \cdots T_{k_{m+1}} \text {. }
\end{aligned}
$$

Note the iterative nature of the identity. The cases $m=0$ and $m=1$ give (2.5) and (3.5), respectively. The case $m=2$ produces

$$
\sum_{\substack{k_{1}+k_{2}+k_{3}+k_{4}=n-2 \\ k_{1}, k_{2}, k_{3}, k_{4} \geq 1}} F_{k_{1}} T_{k_{2}} T_{k_{3}} T_{k_{4}}=\sum_{\substack{k_{1}+k_{2}+k_{3}=n \\ k_{1}, k_{2}, k_{3} \geq 1}} T_{k_{1}} T_{k_{2}} T_{k_{3}}-\sum_{k=1}^{n+2} T_{k} T_{n+2-k}+T_{n+4}-F_{n+4} .
$$

Also, from (4.3) with $u_{n}=L_{n}$ and $v_{n}=T_{n}$ we get

$$
\begin{gathered}
\sum_{\substack{k_{1}+k_{2}+\cdots+k_{m+2}=n-3 \\
k_{1} \geq 0, k_{2}, \ldots, k_{m+2} \geq 1}} L_{k_{1}} T_{k_{2}} \cdots T_{k_{m+2}}=2 \sum_{\substack{k_{1}+k_{2}+\cdots, k_{m+1}=n-1 \\
k_{1}, k_{2}, \ldots, k_{m+1} \geq 1}} T_{k_{1}} T_{k_{2}} \cdots T_{k_{m+1}}-\sum_{\substack{k_{1}+k_{2}+\cdots+k_{m+1}=n \\
k_{1}, k_{2}, \ldots, k_{m+1} \geq 1}} T_{k_{1}} T_{k_{2}} \cdots T_{k_{m+1}} \\
-\sum_{\substack{k_{1}+k_{2}+\cdots+k_{m+1}=n-1 \\
k_{1} \geq 0, k_{2}, \ldots, k_{m+1} \geq 1}} L_{k_{1}} T_{k_{2}} \cdots T_{k_{m+1}} .
\end{gathered}
$$

Again, we point out the iterative nature of the identity. The case $m=0$ gives (2.6). The case $m=1$ is (3.6). The case $m=2$ produces

$$
\begin{aligned}
\sum_{\substack{k_{1}+k_{2}+k_{3}+k_{4}=n-3 \\
k_{1} \geq 0, k_{2}, k_{3}, k_{4} \geq 1}} L_{k_{1}} T_{k_{2}} T_{k_{3}} T_{k_{4}}= & 2 \sum_{\substack{k_{1}+k_{2}+k_{3}=n \\
k_{1}, k_{2}, k_{3} \geq 1}} T_{k_{1}} T_{k_{2}} T_{k_{3}}-\sum_{\substack{k_{1}+k_{2}+k_{3}=n-1 \\
k_{1}, k_{2}, k_{3} \geq 1}} T_{k_{1}} T_{k_{2}} T_{k_{3}} \\
& -\sum_{k=0}^{n+1} T_{k}\left(2 T_{n+2-k}-T_{n+1-k}\right)-T_{n+3}-L_{n+3}+2 T_{n+4} .
\end{aligned}
$$


From (4.4) with $u_{n}=F_{n}$ and $v_{n}=T_{n}$ we obtain

$$
\begin{aligned}
& \sum_{\substack{k_{1}+k_{2}+\cdots+k_{m+2}=n-2 \\
k_{1}, k_{2}, \ldots, k_{m+2} \geq 1}} T_{k_{1}} F_{k_{2}} \cdots F_{k_{m+2}}=\sum_{\substack{k_{1}+k_{2}+\cdots+k_{m+1}=n \\
k_{1}, k_{2}, \ldots, k_{m+1} \geq 1}} T_{k_{1}} F_{k_{2}} \cdots F_{k_{m+1}} \\
& -\sum_{\substack{k_{1}+k_{2}+\cdots+k_{m+1}=n \\
k_{1}, k_{2}, \ldots, k_{m+1} \geq 1}} F_{k_{1}} F_{k_{2}} \cdots F_{k_{m+1}}
\end{aligned}
$$

and with $u_{n}=L_{n}$ and $v_{n}=T_{n}$

$$
\begin{array}{ll}
\sum_{\substack{k_{1}+k_{2}+\cdots+k_{m+2}=n-3 \\
k_{1} \geq 1, k_{2}, \ldots, k_{m+2} \geq 0}} T_{k_{1}} L_{k_{2}} \cdots L_{k_{m+2}}= & 2 \sum_{\substack{k_{1}+k_{2}+\cdots+k_{m+1}=n \\
k_{1} \geq 1, k_{2}, \ldots, k_{m+1} \geq 0}} T_{k_{1}} L_{k_{2}} \cdots L_{k_{m+1}} \\
-\sum_{\substack{k_{1}+k_{2}+\cdots+k_{m+1}=n-1 \\
k_{1}, k_{2}, \ldots, k_{m+1} \geq 0}}\left(T_{k_{1}}+L_{k_{1}}\right) L_{k_{2}} \cdots L_{k_{m+1}} . \\
.
\end{array}
$$

Both results admit an iterative evaluation. We refer to [5] for more details.

In exactly the same manner, we can multiply (2.10) by $f_{v_{2 n}}^{m}(x)$ or $f_{u_{2 n}}^{m}(x)$ to get

$$
\begin{aligned}
u_{0} f_{v_{2 n}}^{m+1}(x)= & v_{0} f_{u_{2 n}}(x) f_{v_{2 n}}^{m}(x)+\left(2 u_{0}-u_{1}\right) x f_{v_{2 n}}^{m+1}(x)+\left(v_{2}-3 v_{0}\right) x f_{u_{2 n}}(x) f_{v_{2 n}}^{m}(x) \\
& +\left(2 v_{1}-v_{2}\right) x^{2} f_{u_{2 n}}(x) f_{v_{2 n}}^{m}(x)+2 x^{2} f_{u_{2 n}}(x) f_{v_{2 n}}^{m+1}(x)+x^{3} f_{u_{2 n}}(x) f_{v_{2 n}}^{m+1}(x)
\end{aligned}
$$

and

$$
\begin{aligned}
v_{0} f_{u_{2 n}}^{m+1}(x)= & u_{0} f_{v_{2 n}}(x) f_{u_{2 n}}^{m}(x)+\left(u_{1}-2 u_{0}\right) x f_{v_{2 n}}(x) f_{u_{2 n}}^{m}(x)+\left(3 v_{0}-v_{2}\right) x f_{u_{2 n}}^{m+1}(x) \\
& +\left(v_{2}-2 v_{1}\right) x^{2} f_{u_{2 n}}^{m+1}(x)-2 x^{2} f_{v_{2 n}}(x) f_{u_{2 n}}^{m+1}(x)-x^{3} f_{v_{2 n}}(x) f_{u_{2 n}}^{m+1}(x) .
\end{aligned}
$$

These formulas lead to the following results:

Theorem 4.2. Let $m \geq 0$ be an integer. Then the following identities hold:

$$
\begin{aligned}
& u_{0} \sum_{k_{1}+k_{2}+\cdots+k_{m+1}=n} v_{2 k_{1}} v_{2 k_{2}} \cdots v_{2 k_{m+1}}=v_{0} \sum_{k_{1}+k_{2}+\cdots+k_{m+1}=n} u_{2 k_{1}} v_{2 k_{2}} \cdots v_{2 k_{m+1}} \\
& +\sum_{k_{1}+k_{2}+\cdots+k_{m+1}=n-1}\left(\left(2 u_{0}-u_{1}\right) v_{2 k_{1}} v_{2 k_{2}} \cdots v_{2 k_{m+1}}+\left(v_{2}-3 v_{0}\right) u_{2 k_{1}} v_{2 k_{2}} \cdots v_{2 k_{m+1}}\right) \\
& +\left(2 v_{1}-v_{2}\right) \sum_{k_{1}+k_{2}+\cdots+k_{m+1}=n-2} u_{2 k_{1}} v_{2 k_{2}} \cdots v_{2 k_{m+1}} \\
& +2 \sum_{k_{1}+k_{2}+\cdots+k_{m+2}=n-2} u_{2 k_{1}} v_{2 k_{2}} \cdots v_{2 k_{m+2}}+\sum_{k_{1}+k_{2}+\cdots+k_{m+2}=n-3} u_{2 k_{1}} v_{2 k_{2}} \cdots v_{2 k_{m+2}}
\end{aligned}
$$


and

$$
\begin{aligned}
& v_{0} \sum_{k_{1}+k_{2}+\cdots+k_{m+1}=n} u_{2 k_{1}} u_{2 k_{2}} \cdots u_{2 k_{m+1}}=u_{0} \sum_{k_{1}+k_{2}+\cdots+k_{m+1}=n} v_{2 k_{1}} u_{2 k_{2}} \cdots u_{2 k_{m+1}} \\
& +\sum_{k_{1}+k_{2}+\cdots+k_{m+1}=n-1}\left(\left(u_{1}-2 u_{0}\right) v_{2 k_{1}} u_{2 k_{2}} \cdots u_{2 k_{m+1}}+\left(3 v_{0}-v_{2}\right) u_{2 k_{1}} u_{2 k_{2}} \cdots u_{2 k_{m+1}}\right) \\
& +\left(v_{2}-2 v_{1}\right) \sum_{k_{1}+k_{2}+\cdots+k_{m+1}=n-2} u_{2 k_{1}} u_{2 k_{2}} \cdots u_{2 k_{m+1}} \\
& \sum_{k_{1}+k_{2}+\cdots+k_{m+2}=n-2} v_{2 k_{1}} u_{2 k_{2}} \cdots u_{2 k_{m+2}}-\sum_{k_{1}+k_{2}+\cdots+k_{m+2}=n-3}
\end{aligned}
$$

When $m=0$, then (4.13) and (4.14) reduce to (2.9). Special cases of (4.13) are

$$
\begin{aligned}
& 2 \sum_{\substack{k_{1}+k_{2}+\cdots+k_{m+2}=n-1 \\
k_{1}, k_{2}, \ldots, k_{m+2} \geq 1}} F_{2 k_{1}} T_{2 k_{2}} \cdots T_{2 k_{m+2}}+\sum_{\substack{k_{1}+k_{2}+\cdots+k_{m+2}=n-2 \\
k_{1}, k_{2}, \ldots, k_{m+2} \geq 1}} F_{2 k_{1}} T_{2 k_{2}} \cdots T_{2 k_{m+2}} \\
& =\sum_{\substack{k_{1}+k_{2}+\cdots+k_{m+1}=n \\
k_{1}, k_{2}, \ldots, k_{m+1} \geq 1}}\left(T_{2 k_{1}}-F_{2 k_{1}}\right) T_{2 k_{2}} \cdots T_{2 k_{m+1}}-\sum_{\substack{k_{1}+k_{2}+\cdots+k_{m+1}=n-1 \\
k_{1}, k_{2}, \ldots, k_{m+1} \geq 1}} F_{2 k_{1}} T_{2 k_{2}} \cdots T_{2 k_{m+1}},
\end{aligned}
$$

and

$$
\begin{aligned}
& 2 \sum_{\substack{k_{1}+k_{2}+\cdots+k_{m+2}=n-2 \\
k_{1} \geq 0, k_{2}, \ldots, k_{m+2} \geq 1}} L_{2 k_{1}} T_{2 k_{2}} \cdots T_{2 k_{m+2}}+\sum_{\substack{k_{1}+k_{2}+\cdots+k_{m+2}=n-3 \\
k_{1} \geq 0, k_{2}, \ldots, k_{m+2} \geq 1}} L_{2 k_{1}} T_{2 k_{2}} \cdots T_{2 k_{m+2}} \\
& =2 \sum_{\substack{k_{1}+k_{2}+\cdots+k_{m+1}=n \\
k_{1}, k_{2}, \ldots, k_{m+1} \geq 1}} T_{2 k_{1}} T_{2 k_{2}} \cdots T_{2 k_{m+1}}-\sum_{\substack{k_{1}+k_{2}+\cdots+k_{m+1}=n-1 \\
k_{1} \geq 0, k_{2}, \ldots, k_{m+1} \geq 1}}\left(3 T_{2 k_{1}}+L_{2 k_{1}}\right) T_{2 k_{2}} \cdots T_{2 k_{m+1}} \\
& -\sum_{\substack{k_{1}+k_{2}+\cdots+k_{m+1}=n-2 \\
k_{1}, k_{2}, \ldots, k_{m+1} \geq 0}} L_{2 k_{1}} L_{2 k_{2}} \cdots L_{2 k_{m+1}} \text {. }
\end{aligned}
$$

Moreover, from (4.14) we obtain

$$
\begin{aligned}
& 2 \sum_{\substack{k_{1}+k_{2}+\cdots+k_{m+2}=n-1 \\
k_{1}, k_{2}, \ldots, k_{m+2} \geq 1}} T_{2 k_{1}} F_{2 k_{2}} \cdots F_{2 k_{m+2}}+\sum_{\substack{k_{1}+k_{2}+\cdots+k_{m+2}=n-2 \\
k_{1}, k_{2}, \ldots, k_{m+2} \geq 1}} T_{2 k_{1}} F_{2 k_{2}} \cdots F_{2 k_{m+2}} \\
& =\sum_{\substack{k_{1}+k_{2}+\cdots+k_{m+1}=n \\
k_{1}, k_{2}, \ldots, k_{m+1} \geq 1}}\left(T_{2 k_{1}}-F_{2 k_{1}}\right) F_{2 k_{2}} \cdots F_{2 k_{m+1}}-\sum_{\substack{k_{1}+k_{2}+\cdots+k_{m+1}=n-1 \\
k_{1}, k_{2}, \ldots, k_{m+1} \geq 1}} F_{2 k_{1}} F_{2 k_{2}} \cdots F_{2 k_{m+1}},
\end{aligned}
$$


and

$$
\begin{aligned}
& 2 \sum_{\substack{k_{1}+k_{2}+\cdots+k_{m+2}=n-2 \\
k_{1} \geq 1, k_{2}, \ldots, k_{m+2} \geq 0}} T_{2 k_{1}} L_{2 k_{2}} \cdots L_{2 k_{m+2}}+\sum_{\substack{k_{1}+k_{2}+\cdots+k_{m+2}=n-3 \\
k_{1} \geq 1, k_{2}, \ldots, k_{m+2} \geq 0}} T_{2 k_{1}} L_{2 k_{2}} \cdots L_{2 k_{m+2}} \\
& =2 \sum_{\substack{k_{1}+k_{2}+\cdots+k_{m+1}=n \\
k_{1} \geq 1, k_{2}, \ldots, k_{m+1} \geq 0}} T_{2 k_{1}} L_{2 k_{2}} \cdots L_{2 k_{m+1}}-\sum_{\substack{k_{1}+k_{2}+\cdots+k_{m+1}=n-1 \\
k_{1}, k_{2}, \ldots, k_{m+1} \geq 0}}\left(3 T_{2 k_{1}}+L_{2 k_{1}}\right) L_{2 k_{2}} \cdots L_{2 k_{m+1}} \\
& -\sum_{\substack{k_{1}+k_{2}+\cdots+k_{m+1}=n-2 \\
k_{1}, k_{2}, \ldots, k_{m+1} \geq 0}} L_{2 k_{1}} L_{2 k_{2}} \cdots L_{2 k_{m+1}} \text {. }
\end{aligned}
$$

The general results for $u_{2 n+1}$ and $v_{2 n+1}$ are derived analogously using (2.16). We leave the explicit statement of these results to the interested reader.

\section{Concluding comments}

The goal of this paper was to develop further identities connecting Fibonacci numbers and Tribonacci numbers. Interestingly, the derivations are based on simple manipulations of the respective generating functions. We hope that the new identities presented in this paper will turn out to be of use to the Fibonacci research community.

We also mention that it seems to be possible to generalize the results of the present study even further to the sequences defined by

$$
U_{n}=p U_{n-1}+q U_{n-2}, \quad U_{0}=a, U_{1}=b, \quad n \geq 2,
$$

and

$$
V_{n}=r V_{n-1}+s V_{n-2}+t V_{n-3}, \quad V_{0}=c, V_{1}=d, V_{2}=e \quad n \geq 3,
$$

where $p, q, a, b, r, s, t, c, d$ and $e$ are arbitrary integers (appropriately restricted to eliminate degenerated cases). The results, however, become cumbersome and bulky.

Finally, the author wants to express his gratitude to the two referees for a careful reading of the first version of the paper, and for the interest shown in this work.

\section{References}

[1] Adegoke, K. (2018). Squares of Tribonacci numbers, Preprint, Available online: https : //arxiv.org/abs/1805.07855v2.

[2] Adegoke, K. (2018). Weighted sums of some second-order sequences, Fibonacci Quart., $56(3), 252-262$.

[3] Cook, C. K.., \& Komatsu, T. (2016). Some identities for sequences of binomial sums of generalized Fibonacci numbers, Fibonacci Quart., 54 (2), 105-111.

[4] Frontczak, R. (2018). Convolutions for generalized Tribonacci numbers and related results, Int. J. Math. Anal., 12 (7), 307-324. 
[5] Frontczak, R. (2018). Some Fibonacci-Lucas-Tribonacci-Lucas identities, Fibonacci Quart., 56 (3), 263-274.

[6] Mezö, I. (2009). Several generating functions for second-order recurrence sequences, $J$. Integer Seq., 12, Article 09.3.7.

[7] Tanackov, I. (2018). Binet type formula for Tribonacci sequence with arbitrary initial numbers, Chaos Solitons Fractals, 114, 63-68.

[8] Sloane, N. J. A. The On-Line Encyclopedia of Integer Sequences, Published electronically: https://oeis.org. 